Journal of Systems Science and Information

Dec., 2016, Vol. 4, No. 6, pp. 574-586

DOI: $10.21078 /$ JSSI-2016-574-13

\title{
Multiattribute Decision Making Method Based on Intuitionistic Linguistic Aggregation Operator
}

\author{
Jing CHEN \\ College of Mathematics and Information Science, Guangxi University, Nanning 530004, China \\ E-mail: chenjing_gxu@sina.com \\ Zhongxing WANG \\ College of Mathematics and Information Science, Guangxi University, Nanning 530004, China
}

\begin{abstract}
In this paper, some new operational laws for intuitionistic linguistic numbers are defined via Archimedean t-norm and s-norm. The prominent feature of these operations is that these operations are closed. Some main properties of these operations, like commutativity, associativity and distribution law, are investigated. Based on these operational laws, intuitionistic linguistic weighted arithmetic averaging operator is given to aggregate intuitionistic linguistic information. Furthermore, in order to reduce uncertain information of intuitionistic linguistic number, hesitancy degree is divided into degrees of membership and non-membership in proportions, and new expected function and score function are built and used to rank intuitionistic linguistic numbers. Finally, an approach is proposed to solve multiattribute decision making problems in which attribute weights are real numbers and attribute values are intuitionistic linguistic numbers, and a real example is provided to show the effectiveness and applicability of the new method.
\end{abstract}

Keywords multiattribute decision making; intuitionistic linguistic number; linguistic term set

\section{Introduction}

Multiattribute decision making (MADM) problems, a significant component of decision theory, have been intensively and extensively focused on. Due to the uncertainty and complexity of MADM problems, attribute values are often suitable to be expressed as fuzzy numbers ${ }^{[1]}$, like interval number ${ }^{[2-4]}$, linguistic variables ${ }^{[5,6]}$, intuitionistic fuzzy number ${ }^{[7-12]}$. As a desirable tool to study MADM problems, the theory of fuzzy set has been rapidly developed and widely used in many aspects, since its birth ${ }^{[10]}$. However, only by membership degree can the fuzzy set reflect the fuzziness. Atanassov ${ }^{[7,9]}$ extended fuzzy sets and presented the notion of intuitionistic fuzzy sets (IFSs) which assign an additional non-membership degree to each element of the fuzzy set. Later, Atanassov and Gargov ${ }^{[8,13]}$ defined interval-valued intuitionistic fuzzy sets (IVIFSs), its striking characteristics lies in a fact that the membership degree and nonmembership degree are both interval numbers instead of certain numbers. Shu and Cheng ${ }^{[14]}$ presented the concept of the intuitionistic triangle fuzzy number. And with the membership degree and the non-membership degree being denoted by triangular fuzzy numbers, Chen and

Received March 10, 2016, accepted April 23, 2016

Supported by the National Natural Science Foundation of China (71261001) 
$\mathrm{Li}^{[15]}$ defined the triangular intuitionistic fuzzy number (TIFN) and proposed the weighted arithmetic averaging operator on TIFNs.

However, the membership degree and non-membership degree to a particular fuzzy linguistic index become major considerations for IFSs, IVIFSs, ITFNs and TIFNs, and a single fuzzy linguistic index can't accurately express the evaluation information for an object. Moreover, in many practical cases, the available decision information is usually difficult to be judged precisely; instead, it can be easily characterized by some fuzzy linguistic terms, such as "good", "average", "poor", etc. Wang and $\mathrm{Li}^{[16,17]}$ generalized fuzzy linguistic term set and gave the definition of intuitionistic linguistic set, which more flexible and practical than linguistic term set in dealing with fuzziness and uncertainty. Liu ${ }^{[18]}$ developed two generalized dependent aggregation operators for intuitionistic linguistic numbers and investigated some main properties of these operators. Similarly, based on the uncertain linguistic variable, Liu and Jin ${ }^{[19]}$ further proposed intuitionistic uncertain linguistic variable (IULV) and introduced some operational laws, two continuous ranking function of IULVs and three geometric operators. Inspired by Atanassov's IVIFSs, $\mathrm{Liu}^{[20]}$ generalized the concepts of IULV to interval-valued intuitionistic uncertain linguistic variable (IVIULV) and presented the weighted geometric average (WGA) operator, the ordered weighted geometric (OWG) operator and the hybrid geometric (HG) operator with IVIULVs. After that, Meng and Chen ${ }^{[21]}$ defined the Choquet averaging (IVIULCA) operator and the Choquet geometric mean (IVIULCGM) operator with IVIULVs.

Nevertheless, some operational laws for intuitionistic linguistic numbers defined by Wang ${ }^{[22]}$ and Liu ${ }^{[18,23]}$ are not closed. In order to overcome the blemish, Archimedean t-norm and snorm have been employed to deal with the intuitionistic linguistic information in this paper. To do this, the rest of this paper is organized as follows: In Section 2, we will briefly introduce some basic concepts. In Section 3, some new operational laws will be defined via Archimedean tnorm and s-norm. Furthermore, some desirable properties of these operations and some special cases are studied. Later, accumulated expectation function and accumulated score function are introduced for ranking intuitionistic linguistic numbers. In Section 4, a new ILWAA operator is developed. In Section 5, a new method to MADM problems with intuitionistic linguistic information is generated on the basis of ILWAA operator. At last, an illustrative example was given to demonstrate the practicality and effectiveness of the developed method.

\section{Preliminaries}

In this section, the concepts of Archimedean t-norm, Archimedean s-norm, linguistic term sets and intuitionistic linguistic sets will be reviewed.

\subsection{Triangular Norms and Triangular Conorms}

Definition 1 (see [24]) A real-valued function $T:[0,1] \times[0,1] \rightarrow[0,1]$ is named as a triangular norm (t-norm for short), if $T$ satisfies the following four conditions:

1) (Commutativity) $T(x, y)=T(y, x)$, for all $x, y \in[0,1]$.

2) (Associativity) $T(T(x, y), z)=T(x, T(y, z))$, for all $x, y, z \in[0,1]$.

3) (Monotonicity) $T(x, y) \leq T\left(x^{\prime}, y^{\prime}\right)$, if $0 \leq x \leq x^{\prime} \leq 1,0 \leq y \leq y^{\prime} \leq 1$. 
4) (Boundary condition) $T(1, x)=x$, for all $x \in[0,1]$.

Definition 2 (see [24]) A real-valued function $S:[0,1] \times[0,1] \rightarrow[0,1]$ is named as a triangular conorm (s-norm for short), if $S$ satisfies the following four conditions:

1) (Commutativity) $S(x, y)=S(y, x)$, for all $x, y \in[0,1]$.

2) (Associativity) $S(S(x, y), z)=S(x, S(y, z))$, for all $x, y, z \in[0,1]$.

3) (Monotonicity) $S(x, y) \leq S\left(x^{\prime}, y^{\prime}\right)$, if $0 \leq x \leq x^{\prime} \leq 1,0 \leq y \leq y^{\prime} \leq 1$.

4) (Boundary condition) $S(0, x)=x$, for all $x \in[0,1]$.

It is well known that s-norm and t-norm are the dual ${ }^{[24]}$. In other words, there exists a decreasing function $N:[0,1] \rightarrow[0,1]$ with $N(0)=1$ and $N(1)=0$, such that

$$
T(x, y)=S(N(x), N(y)) .
$$

Especially, if $N(t)=1-t$, then $T(x, y)=S(1-x, 1-y)$.

Definition 3 (see [24]) A t-norm $T(x, y)$ is called Archimedean t-norm, if it is continuous and $T(x, x)<x$, for each $x \in(0,1)$.

Definition 4 (see [24]) An s-norm $S(x, y)$ is called Archimedean s-norm, if it is continuous and $S(x, x)>x$, for each $x \in(0,1)$.

Theorem 1 Let $g:(0,1] \rightarrow[0,+\infty)$ be a strictly monotone decreasing and continuous function such that $g(1)=0$ and $g(0)=+\infty$, if $N(t)=1-t$ and $f(t)=g(N(t))$, then Archimedean t-norm and its dual s-norm could be represented as

$$
T(x, y)=g^{-1}(g(x)+g(y)), \quad S(x, y)=f^{-1}(f(x)+f(y)),
$$

where $g^{-1}(t)$ and $f^{-1}(t)$ denote the inverse function of $g(t)$ and $f(t)$, respectively.

Proof We want to show that $T(x, y)=g^{-1}(g(x)+g(y))$ is an Archimedean t-norm, it suffices to prove that the function $T:[0,1] \times[0,1] \rightarrow[0,1]$ is a t-norm and $T(x, x)=g^{-1}(g(x)+$ $g(x))<x$, for each $x \in(0,1)$. We proceed stepwise.

1) $T(x, y)=g^{-1}(g(x)+g(y))=g^{-1}(g(y)+g(x))=T(y, x)$.

2) $\quad T(x, T(y, z))$

$=g^{-1}\left(g(x)+g\left(g^{-1}(g(y)+g(z))\right)\right)$

$=g^{-1}(g(x)+g(y)+g(z))$

$=g^{-1}\left(g\left(g^{-1}(g(x)+g(y))\right)+g(z)\right)$

$=T(T(x, y), z)$.

3) Since the inverse function $g^{-1}$ is a strictly monotone decreasing and continuous function, if $x \leq x^{\prime}$ and $y \leq y^{\prime}$, then $g(x)+g(y) \geq g\left(x^{\prime}\right)+g\left(y^{\prime}\right)$, and thus,

$$
g^{-1}(g(x)+g(y)) \leq g^{-1}\left(g\left(x^{\prime}\right)+g\left(y^{\prime}\right)\right),
$$

that is,

$$
T(x, y) \leq T\left(x^{\prime}, y^{\prime}\right)
$$


4) Since $g(1)=0$, then $T(1, x)=g^{-1}(g(1)+g(x))=g^{-1}(0+g(x))=x$.

5) Especially, if $x \in(0,1)$, then

$$
T(x, x)=g^{-1}(g(x)+g(x))<g^{-1}(g(x)+g(1))=x .
$$

According to Definition 1 and Definition 3, we prove that $T(x, y)=g^{-1}(g(x)+g(y))$ is an Archimedean t-norm. The same reasoning also can be applied to prove that $S(x, y)=$ $f^{-1}(f(x)+f(y))$ is an Archimedean s-norm. The proof is omitted.

\subsection{The Linguistic Term Set and Its Extension}

A linguistic term set $L_{2 \tau}=\left\{l_{0}, l_{1}, \cdots, l_{2 \tau}\right\}$ is a finite and totally ordered discrete term set, where $l_{i}$ represents a predefined linguistic term and $\tau$ is a positive integer. For instance, a linguistic term set of five terms can be given by $L_{4}=\left\{l_{0}\right.$ (extremely poor), $l_{1}$ (poor), $l_{2}$ (average), $l_{3}$ (good), $l_{4}$ (extremely good) $\}$. For any linguistic term, it is usually required that there exist the following characteristics ${ }^{[25]}$ :

1) The set is ordered: $l_{i} \preceq l_{j}$, if and only if $i \leq j$.

2) The negation operator is defined: $\operatorname{neg}\left(l_{i}\right)=l_{2 \tau-i}$.

3) The max operator: $\max \left\{l_{i}, l_{j}\right\}=l_{j}$, if $l_{i} \preceq l_{j}$.

4) The min operator: $\min \left\{l_{i}, l_{j}\right\}=l_{i}$, if $l_{i} \preceq l_{j}$.

Generally, in the process of aggregating information, the calculated result might not be contained in the predefined linguistic term set $L_{2 \tau}$, which often results in loss of information. In order to preserve all the given information, Herrera ${ }^{[26]}$ extended the discrete linguistic term set $L_{2 \tau}=\left\{l_{0}, l_{1}, \cdots, l_{2 \tau}\right\}$ to a continuous linguistic term set $\widetilde{L_{2 \tau}}=\left\{l_{\alpha} \mid \alpha \in[0,2 \tau]\right\}$, where if $l_{\alpha} \in L_{2 \tau}$, we then call $l_{\alpha}$ an original linguistic term; otherwise, we call $l_{\alpha}$ an extended linguistic term. The extended linguistic terms also satisfies the above characteristics.

Given two linguistic terms $l_{\alpha_{1}}$ and $l_{\alpha_{2}}$, some operational laws are expressed as follows ${ }^{[27-29]}$ :

1) $l_{\alpha_{1}} \oplus l_{\alpha_{2}}=l_{\alpha_{1}+\alpha_{2}}$.

2) $k l_{\alpha_{1}}=l_{k \alpha_{1}}, k \geq 0$.

\subsection{The Intuitionistic Linguistic Set}

Wang ${ }^{[16,22]}$ generalized linguistic term set and presented the concept of intuitionistic linguistic set.

Definition 5 (see $[16,18]$ ) Let $L_{2 \tau}$ be a linguistic term set, an intuitionistic linguistic set (ILS) $H$ in $X$ is defined as

$$
H=\left\{\left\langle x,\left[l_{\theta(x)}, u(x), v(x)\right]\right\rangle \mid x \in X\right\} .
$$

Here $l_{\theta(x)} \in L_{\tau}, u: X \rightarrow[0,1]$ and $v: X \rightarrow[0,1]$, with the condition $0 \leq u(x)+v(x) \leq 1$. The numbers $u(x)$ and $v(x)$ represent, respectively, the membership degree and non-membership degree of the element $x$ to term $l_{\theta(x)}$. 
For each ILS $H$ in $X$, let $\pi(x)=1-u(x)-v(x), \forall x \in X$, we then call $\pi(x)$ a hesitancy degree of $x$ to linguistic term $l_{\theta(x)}$. It is obvious that $0 \leq \pi(x) \leq 1, \forall x \in X$.

Definition 6 (see $[16,18]$ ) Let $H=\left\{\left\langle x,\left[l_{\theta(x)}, u(x), v(x)\right]\right\rangle \mid x \in X\right\}$ be an ILS, we then call the ternary group $\left\langle l_{\theta(x)}, u(x), v(x)\right\rangle$ an intuitionistic linguistic number (ILN), and $H$ can also be regarded as a set of ILNs. Thus, we can denote ILS by $H=\left\{\left\langle l_{\theta(x)}, u(x), v(x)\right\rangle \mid x \in X\right\}$.

Given two ILNs $h_{1}=\left\langle l_{\theta\left(h_{1}\right)}, u\left(h_{1}\right), v\left(h_{1}\right)\right\rangle$ and $h_{2}=\left\langle l_{\theta\left(h_{2}\right)}, u\left(h_{2}\right), v\left(h_{2}\right)\right\rangle$, some operational laws are expressed as follows ${ }^{[16,18]}$ :

1) $h_{1} \oplus h_{2}=\left\langle l_{\theta\left(h_{1}\right)+\theta\left(h_{2}\right)}, u\left(h_{1}\right)+u\left(h_{2}\right)-u\left(h_{1}\right) u\left(h_{2}\right), v\left(h_{1}\right) v\left(h_{1}\right)\right\rangle$.

2) $k h_{1}=\left\langle l_{k \theta\left(h_{1}\right)}, 1-\left[1-u\left(h_{1}\right)\right]^{k}, v\left(h_{1}\right)^{k}\right\rangle, k \geq 0$.

3) $h_{1} \otimes h_{2}=\left\langle l_{\theta\left(h_{1}\right) \times \theta\left(h_{2}\right)}, u\left(h_{1}\right) u\left(h_{1}\right), v\left(h_{1}\right)+v\left(h_{2}\right)-v\left(h_{1}\right) v\left(h_{2}\right)\right\rangle$.

4) $h_{1}{ }^{k}=\left\langle l_{k \theta\left(h_{1}\right)}, u\left(h_{1}\right)^{k}, 1-\left[1-v\left(h_{1}\right)\right]^{k}\right\rangle, k \geq 0$.

These operations are widely used in MADM problems with intuitionistic linguistic information to produce the calculated results of attribute values. However, as the following example illustrates, the calculated results might not match any element in ILS.

Example 1 Let $L_{4}=\left\{l_{0}\right.$ (very poor), $l_{1}$ (poor), $l_{2}$ (average), $l_{3}$ (good), $l_{4}($ verygood $\left.)\right\}$ be a linguistic term set and $H=\left\{\left\langle x,\left[l_{\theta(x)}, u_{H}(x), v_{H}(x)\right]\right\rangle \mid x \in X\right\}$ be an ILS, if we apply these operations $^{[16,18]}$ for $\left\langle l_{1}, 0.5,0.2\right\rangle,\left\langle l_{3}, 0.6,0.2\right\rangle$ and $\left\langle l_{4}, 0.1,0.7\right\rangle$, then we have the following results:

$$
\begin{aligned}
& \left\langle l_{1}, 0.5,0.2\right\rangle \oplus\left\langle l_{3}, 0.6,0.2\right\rangle=\left\langle l_{4}, 0.8,0.04\right\rangle, \\
& \left\langle l_{1}, 0.5,0.2\right\rangle \otimes\left\langle l_{3}, 0.6,0.2\right\rangle=\left\langle l_{3}, 0.3,0.36\right\rangle, \\
& \left\langle l_{3}, 0.6,0.2\right\rangle \oplus\left\langle l_{4}, 0.1,0.7\right\rangle=\left\langle l_{7}, 0.64,0.14\right\rangle(\notin H), \\
& \left\langle l_{3}, 0.6,0.2\right\rangle \otimes\left\langle l_{4}, 0.1,0.7\right\rangle=\left\langle l_{12}, 0.06,0.86\right\rangle(\notin H) .
\end{aligned}
$$

Obviously, the linguistic term " $l_{7}$ " and " $l_{12}$ " in the calculated results don't belong to the linguistic term set $L_{4}$, and the calculated result of the ILNs $\left\langle l_{3}, 0.6,0.2\right\rangle$ and $\left\langle l_{4}, 0.1,0.7\right\rangle$ is not successful. Besides, formula (4) shows that the calculated result of the linguistic term "very poor" and "good" is "very good". This is not in accordance with actual situations. In fact, one term between "very poor" and "good" may be more easily accepted.

To avoid the above-mentioned problems, we shall give another kind of operational laws to solve these problems existed.

\section{New Operations for Intuitionistic Linguistic Numbers}

\subsection{ILS's Extension and New Operations}

Similarity to the definition of the extended linguistic term set, we define the extended intuitionistic linguistic set. And based on the extended intuitionistic linguistic set and Archimedean t-norm, Archimedean s-norm, we define some new operational laws.

Definition 7 Let $X$ be a given domain, and $\widetilde{L_{2 \tau}}=\left\{l_{\alpha} \mid \alpha \in[0,2 \tau]\right\}$ be the extension of a linguistic term set $L_{2 \tau}=\left\{l_{0}, l_{1}, \cdots, l_{2 \tau}\right\}$, then

$$
\widetilde{H}=\left\{\left\langle l_{\eta(x)}, u(x), v(x)\right\rangle \mid x \in X, l_{\eta(x)} \in \widetilde{L_{2 \tau}}\right\}
$$


is named as an extension of $H=\left\{\left\langle l_{\theta(x)}, u(x), v(x)\right\rangle \mid x \in X, l_{\theta(x)} \in L_{2 \tau}\right\}$.

Based on the extented ILS $\widetilde{H}$, some new operational laws for ILNs can be shown as follows.

Definition 8 Let $h_{1}=\left\langle l_{\eta\left(h_{1}\right)}, u\left(h_{1}\right), v\left(h_{1}\right)\right\rangle$ and $h_{2}=\left\langle l_{\eta\left(h_{2}\right)}, u\left(h_{2}\right), v\left(h_{2}\right)\right\rangle$ be any two ILNs, and $k \geq 0$ be a scalar, then new operations are defined as

1) $h_{1} \oplus h_{2}=\left\langle l_{\omega_{2}\left(h_{1}\right) \eta\left(h_{1}\right)+\omega_{2}\left(h_{2}\right) \eta\left(h_{2}\right)}, f^{-1}\left(\sum_{i=1}^{2} f\left(u\left(h_{i}\right)\right)\right), g^{-1}\left(\sum_{i=1}^{2} g\left(v\left(h_{i}\right)\right)\right)\right\rangle$.

2) $k h_{1}=\left\langle l_{\eta\left(h_{1}\right)}, f^{-1}\left(k f\left(u\left(h_{1}\right)\right)\right), g^{-1}\left(k g\left(v\left(h_{1}\right)\right)\right)\right\rangle$.

3) $h_{1} \otimes h_{2}=\left\langle l_{\varpi_{2}\left(h_{1}\right) \eta\left(h_{1}\right)+\varpi_{2}\left(h_{2}\right) \eta\left(h_{2}\right)}, g^{-1}\left(\sum_{i=1}^{2} g\left(u\left(h_{i}\right)\right)\right), f^{-1}\left(\sum_{i=1}^{2} f\left(v\left(h_{i}\right)\right)\right)\right\rangle$.

4) $h_{1}^{k}=\left\langle l_{\eta\left(h_{1}\right)}, g^{-1}\left(k g\left(u\left(h_{1}\right)\right)\right), f^{-1}\left(k f\left(v\left(h_{1}\right)\right)\right)\right\rangle$.

Note that $\sum_{i=1}^{2} \omega_{2}\left(h_{i}\right) \eta\left(h_{i}\right)$ and $\sum_{i=1}^{2} \varpi_{2}\left(h_{i}\right) \eta\left(h_{i}\right)$ are different convex combination of $\eta\left(h_{i}\right)(i=1,2)$ such that

$$
\omega_{2}\left(h_{i}\right)=\frac{f\left(u\left(h_{i}\right)\right)+g\left(v\left(h_{i}\right)\right)}{\sum_{i=1}^{2} f\left(u\left(h_{i}\right)\right)+g\left(v\left(h_{i}\right)\right)}, \quad \varpi_{2}\left(h_{i}\right)=\frac{g\left(u\left(h_{i}\right)\right)+f\left(v\left(h_{i}\right)\right)}{\sum_{i=1}^{2} g\left(u\left(h_{i}\right)\right)+f\left(v\left(h_{i}\right)\right)},
$$

where $g:(0,1] \rightarrow[0,+\infty)$ is a strictly monotone decreasing and continuous function, which satisfies $g(0)=+\infty$ and $g(1)=0$, and $f(x)=g(1-x)$.

Theorem 2 Suppose that $g(t)=-\log t, f(t)=-\log (1-t)$, Definition 8 can be re-written as

1) $h_{1} \oplus h_{2}=\left\langle l_{\sum_{i=1}^{2} \frac{\eta\left(h_{i}\right) \ln \left[\left(1-u\left(h_{i}\right)\right) v\left(h_{i}\right)\right]}{\sum_{i=1}^{i} \ln \left[\left(1-u\left(h_{i}\right)\right) v\left(h_{i}\right)\right]}} u\left(h_{1}\right)+u\left(h_{2}\right)-u\left(h_{1}\right) u\left(h_{2}\right), v\left(h_{1}\right) v\left(h_{1}\right)\right\rangle$.

2) $k h_{1}=\left\langle l_{\eta\left(h_{1}\right)}, 1-\left[1-u\left(h_{1}\right)\right]^{k}, v\left(h_{1}\right)^{k}\right\rangle$.

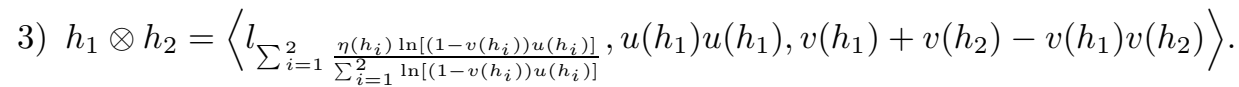

4) $h_{1}^{k}=\left\langle l_{\eta\left(h_{1}\right)}, u\left(h_{1}\right)^{k}, 1-\left[1-v\left(h_{1}\right)\right]^{k}\right\rangle$.

Example 2 For Example 1, applying Theorem 2 for $\left\langle l_{1}, 0.5,0.2\right\rangle,\left\langle l_{3}, 0.6,0.2\right\rangle$ and $\left\langle l_{4}, 0.1,0.7\right\rangle$, we can get

$$
\begin{aligned}
& \left\langle l_{1}, 0.5,0.2\right\rangle \oplus\left\langle l_{3}, 0.6,0.2\right\rangle=\left\langle l_{2}, 0.8,0.04\right\rangle, \\
& \left\langle l_{1}, 0.5,0.2\right\rangle \otimes\left\langle l_{3}, 0.6,0.2\right\rangle=\left\langle l_{2}, 0.3,0.36\right\rangle, \\
& \left\langle l_{3}, 0.6,0.2\right\rangle \oplus\left\langle l_{4}, 0.1,0.7\right\rangle=\left\langle l_{3}, 0.64,0.14\right\rangle, \\
& \left\langle l_{3}, 0.6,0.2\right\rangle \otimes\left\langle l_{4}, 0.1,0.7\right\rangle=\left\langle l_{3}, 0.06,0.86\right\rangle .
\end{aligned}
$$

Making a comparision between Example 1 and Example 2 shows that the results deduced from new operations are more suitable for actual situation.

Additionally, we can give some properties of these new operations.

Proposition 1 Let $h, h_{1}, h_{2}$ be any three ILNs and $k, k_{1}, k_{2} \geq 0$, new operations satisfies the following properties:

1) $h_{1} \oplus h_{2}=h_{2} \oplus h_{1}$. 
2) $h_{1} \otimes h_{2}=h_{2} \otimes h_{1}$.

3) $k\left(h_{1} \oplus h_{2}\right)=k h_{1} \oplus k h_{2}$.

4) $\left(h_{1} \otimes h_{2}\right)^{k}=h_{1}{ }^{k} \otimes h_{2}{ }^{k}$.

5) $k_{1} h \oplus k_{2} h=\left(k_{1}+k_{2}\right) h$.

6) $h^{k_{1}} \otimes h^{k_{2}}=h^{\left(k_{1}+k_{2}\right)}$.

7) $h_{1} \oplus h_{2} \in \widetilde{H}, k h_{1} \in \widetilde{H}$.

8) $h_{1} \otimes h_{2} \in \widetilde{H}, h_{1}{ }^{k} \in \widetilde{H}$.

Proof 1), 2) According to Definition 8, properties 1) and 2) are correct.

3)

$$
\begin{aligned}
& k\left(h_{1} \oplus h_{2}\right) \\
= & k\left\langle l_{\omega_{2}\left(h_{1}\right) \eta\left(h_{1}\right)+\omega_{2}\left(h_{2}\right) \eta\left(h_{2}\right)}, f^{-1}\left(\sum_{i=1}^{2} f\left(u\left(h_{i}\right)\right)\right), g^{-1}\left(\sum_{i=1}^{2} g\left(v\left(h_{i}\right)\right)\right)\right\rangle \\
= & k\left\langle l_{\frac{\sum_{i=1}^{2}\left[f\left(u\left(h_{i}\right)\right)+g\left(v\left(h_{i}\right)\right)\right] \eta\left(h_{i}\right)}{\sum_{i=1}^{2} f\left(u\left(h_{i}\right)\right)+g\left(v\left(h_{i}\right)\right)}}, f^{-1}\left(\sum_{i=1}^{2} f\left(u\left(h_{i}\right)\right)\right), g^{-1}\left(\sum_{i=1}^{2} g\left(v\left(h_{i}\right)\right)\right)\right\rangle \\
= & \left\langle l_{\frac{\sum_{i=1}^{2}\left[f\left(u\left(h_{i}\right)\right)+g\left(v\left(h_{i}\right)\right)\right] \eta\left(h_{i}\right)}{\sum_{i=1}^{2} f\left(u\left(h_{i}\right)\right)+g\left(v\left(h_{i}\right)\right)}}, f^{-1}\left(\sum_{i=1}^{2} k f\left(u\left(h_{i}\right)\right)\right), g^{-1}\left(\sum_{i=1}^{2} k g\left(v\left(h_{i}\right)\right)\right)\right\rangle \\
= & \left\langle l_{\omega_{2}\left(h_{1}\right) \eta\left(h_{1}\right)+\omega_{2}\left(h_{2}\right) \eta\left(h_{2}\right)}, f^{-1}\left(\sum_{i=1}^{2} k f\left(u\left(h_{i}\right)\right)\right), g^{-1}\left(\sum_{i=1}^{2} k g\left(v\left(h_{i}\right)\right)\right)\right\rangle \\
= & k h_{1} \oplus k h_{2} .
\end{aligned}
$$

4)

$$
\begin{aligned}
& \left(h_{1} \otimes h_{2}\right)^{k} \\
= & \left\langle l_{\varpi_{2}\left(h_{1}\right) \eta\left(h_{1}\right)+\varpi_{2}\left(h_{2}\right) \eta\left(h_{2}\right)}, g^{-1}\left(\sum_{i=1}^{2} g\left(u\left(h_{i}\right)\right)\right), f^{-1}\left(\sum_{i=1}^{2} f\left(v\left(h_{i}\right)\right)\right)\right\rangle^{k} \\
= & \left\langle l_{\frac{\sum_{i=1}^{2}\left[g\left(u\left(h_{i}\right)\right)+f\left(v\left(h_{i}\right)\right)\right] \eta\left(h_{i}\right)}{\sum_{i=1}^{2} g\left(u\left(h_{i}\right)\right)+f\left(v\left(h_{i}\right)\right)}}, g^{-1}\left(\sum_{i=1}^{2} g\left(u\left(h_{i}\right)\right)\right), f^{-1}\left(\sum_{i=1}^{2} f\left(v\left(h_{i}\right)\right)\right)\right\rangle^{k} \\
= & \left\langle l_{\frac{\sum_{i=1}^{2}\left[g\left(u\left(h_{i}\right)\right)+f\left(v\left(h_{i}\right)\right)\right] \eta\left(h_{i}\right)}{\sum_{i=1}^{2} g\left(u\left(h_{i}\right)\right)+f\left(v\left(h_{i}\right)\right)}}, g^{-1}\left(\sum_{i=1}^{2} k g\left(u\left(h_{i}\right)\right)\right), f^{-1}\left(\sum_{i=1}^{2} k f\left(v\left(h_{i}\right)\right)\right)\right\rangle \\
= & \left\langle l_{\varpi_{2}\left(h_{1}\right) \eta\left(h_{1}\right)+\varpi_{2}\left(h_{2}\right) \eta\left(h_{2}\right)}, g^{-1}\left(\sum_{i=1}^{2} k g\left(u\left(h_{i}\right)\right)\right), f^{-1}\left(\sum_{i=1}^{2} k f\left(v\left(h_{i}\right)\right)\right)\right\rangle \\
= & h_{1}^{k} \otimes h_{2}^{k} .
\end{aligned}
$$


5)

$$
\begin{aligned}
& k_{1} h \oplus k_{2} h \\
= & \left\langle l_{\eta(h)}, f^{-1}\left(k_{1} f(u(h))\right), g^{-1}\left(k_{1} g(v(h))\right)\right\rangle \oplus\left\langle l_{\eta(h)}, f^{-1}\left(k_{2} f(u(h))\right), g^{-1}\left(k_{2} g(v(h))\right)\right\rangle \\
= & \left\langle l_{\eta(h)}, f^{-1}\left(k_{1} f(u(h))+k_{2} f(u(h))\right), g^{-1}\left(k_{1} g(v(h))+k_{2} g(v(h))\right)\right\rangle \\
= & \left(k_{1}+k_{2}\right) h .
\end{aligned}
$$

6)

$$
\begin{aligned}
& h^{k_{1}} \oplus h^{k_{2}}=\left\langle l_{\eta(h)}, g^{-1}\left(k_{1} g(u(h))\right), f^{-1}\left(k_{1} f(v(h))\right)\right\rangle \oplus\left\langle l_{\eta(h)}, g^{-1}\left(k_{2} g(u(h))\right),\right. \\
& \begin{aligned}
\left.f^{-1}\left(k_{2} f(v(h))\right)\right\rangle & =\left\langle l_{\eta(h)}, g^{-1}\left(k_{1} g(u(h))+k_{2} g(u(h))\right), f^{-1}\left(k_{1} f(v(h))+k_{2} f(v(h))\right)\right\rangle \\
& =h^{\left(k_{1}+k_{2}\right)} .
\end{aligned}
\end{aligned}
$$

7) Let $h_{1}=\left\langle l_{\eta}\left(h_{1}\right), u\left(h_{1}\right), v\left(h_{1}\right)\right\rangle$ and $h_{2}=\left\langle l_{\eta}\left(h_{2}\right), u\left(h_{2}\right), v\left(h_{2}\right)\right\rangle \in \widetilde{H}$, where

$$
\left\{\begin{array}{l}
0 \leq u\left(h_{1}\right), v\left(h_{1}\right) \leq 1, \\
0 \leq u\left(h_{1}\right)+v\left(h_{1}\right) \leq 1,
\end{array}\right.
$$

and

$$
\left\{\begin{array}{l}
0 \leq u\left(h_{2}\right), v\left(h_{2}\right) \leq 1, \\
0 \leq u\left(h_{2}\right)+v\left(h_{2}\right) \leq 1 .
\end{array}\right.
$$

It is known that $f(x)=g(1-x)$, and $g:(0,1] \rightarrow[0,+\infty)$ is a strictly monotone decreasing which indicates that

$$
0 \leq f^{-1}\left(f\left(u\left(h_{1}\right)\right)+f\left(u\left(h_{2}\right)\right)\right) \leq 1,0 \leq g^{-1}\left(g\left(v\left(h_{1}\right)\right)+g\left(v\left(h_{2}\right)\right)\right) \leq 1,
$$

and

$$
0 \leq f^{-1}\left(f\left(u\left(h_{1}\right)\right)+f\left(u\left(h_{2}\right)\right)\right)+g^{-1}\left(g\left(v\left(h_{1}\right)\right)+g\left(v\left(h_{2}\right)\right)\right) \leq 1 .
$$

In addition, $\eta\left(h_{1}\right), \eta\left(h_{2}\right) \in[0,2 \tau]$, it follows that

$$
0 \leq \omega_{2}\left(h_{1}\right) \eta\left(h_{1}\right)+\omega_{2}\left(h_{2}\right) \eta\left(h_{2}\right) \leq 2 \tau .
$$

Formula (16) along with formula (14) and formula (15) shows that $h_{1} \oplus h_{2} \in \widetilde{H}$ is correct. The rest of rules 7 ) readily follows.

8) The proof of property 8) is similar to that of property 7) and thus is omitted.

Definition 9 Let $h=\left\langle l_{\eta(h)}, u(h), v(h)\right\rangle$ be an ILN, we then call $u_{E}(h)$ and $v_{E}(h)$, respectively, accumulated membership degree and accumulated non-membership degree, if

$$
\begin{aligned}
& u_{E}(h)=u(h)+u(h) \pi(h)+u(h) \pi^{2}(h)+\cdots=\frac{u(h)}{1-\pi(h)}, \\
& v_{E}(h)=v(h)+v(h) \pi(h)+v(h) \pi^{2}(h)+\cdots=\frac{v(h)}{1-\pi(h)} .
\end{aligned}
$$


Definition 10 Let $h=\left\langle l_{\eta(h)}, u(h), v(h)\right\rangle$ be an ILN, $u_{E}(h)$ be accumulated membership degree and $v_{E}(h)$ be accumulated non-membership degree, we then call $E(h)$ and $S(h)$, respectively, accumulated expectation function and accumulated score function, if

$$
\begin{aligned}
& E(h)=u_{E}(h) \eta(h), \\
& S(h)=\left[u_{E}(h)-v_{E}(h)\right] \eta(h) .
\end{aligned}
$$

Definition 11 Let $h_{1}$ and $h_{2}$ be any two ILNs, then

1) If $E\left(h_{1}\right)<E\left(h_{2}\right)$, then $h_{1} \prec h_{2}$.

2) If $E\left(h_{1}\right)=E\left(h_{2}\right)$, then

(a) when $S\left(h_{1}\right)<S\left(h_{2}\right)$, then $h_{1} \prec h_{2}$.

(b) when $S\left(h_{1}\right)=S\left(h_{2}\right)$, then $h_{1}=h_{2}$.

\subsection{Extended Operations for Intuitionistic Linguistic Numbers}

Definition 12 Let $h_{i}=\left\langle l_{\eta\left(h_{i}\right)}, u\left(h_{i}\right), v\left(h_{i}\right)\right\rangle(i=1,2, \cdots, n)$ be a collection of ILNs and $k \geq 0$ be a scalar, then extended operations are defined as

1)

$$
\begin{aligned}
& h_{1} \oplus h_{2} \oplus \cdots \oplus h_{n} \\
= & \left\langle l_{\sum_{i=1}^{n} \omega_{2}\left(h_{i}\right) \eta\left(h_{i}\right)}, f^{-1}\left(\sum_{i=1}^{n} f\left(u\left(h_{i}\right)\right)\right), g^{-1}\left(\sum_{i=1}^{n} g\left(v\left(h_{i}\right)\right)\right)\right\rangle .
\end{aligned}
$$

2)

$$
k h_{1}=\left\langle l_{\eta\left(h_{1}\right)}, f^{-1}\left(k f\left(u\left(h_{1}\right)\right)\right), g^{-1}\left(k g\left(v\left(h_{1}\right)\right)\right)\right\rangle .
$$

3)

$$
\begin{aligned}
& h_{1} \otimes h_{2} \otimes \cdots \otimes h_{n} \\
= & \left\langle l_{\sum_{i=1}^{n} \varpi_{2}\left(h_{i}\right) \eta\left(h_{i}\right)}, g^{-1}\left(\sum_{i=1}^{n} g\left(u\left(h_{i}\right)\right)\right), f^{-1}\left(\sum_{i=1}^{n} f\left(v\left(h_{i}\right)\right)\right)\right\rangle .
\end{aligned}
$$

4)

$$
h_{1}^{k}=\left\langle l_{\eta\left(h_{1}\right)}, g^{-1}\left(k g\left(u\left(h_{1}\right)\right)\right), f^{-1}\left(k f\left(v\left(h_{1}\right)\right)\right)\right\rangle .
$$

Note that $\sum_{i=1}^{n} \omega_{2}\left(h_{i}\right) \eta\left(h_{i}\right)$ and $\sum_{i=1}^{n} \varpi_{2}\left(h_{i}\right) \eta\left(h_{i}\right)$ are different convex combination of $\eta\left(h_{i}\right)(i=1,2, \cdots, n)$ such that

$$
\omega_{n}\left(h_{i}\right)=\frac{f\left(u\left(h_{i}\right)\right)+g\left(v\left(h_{i}\right)\right)}{\sum_{i=1}^{n} f\left(u\left(h_{i}\right)\right)+g\left(v\left(h_{i}\right)\right)}, \quad \varpi_{n}\left(h_{i}\right)=\frac{g\left(u\left(h_{i}\right)\right)+f\left(v\left(h_{i}\right)\right)}{\sum_{i=1}^{n} g\left(u\left(h_{i}\right)\right)+f\left(v\left(h_{i}\right)\right)} .
$$

Definition 13 Let ILWAA : $\widetilde{H}^{n} \rightarrow \widetilde{H}$, if

$$
\begin{aligned}
& \operatorname{ILWAA}\left(h_{1}, h_{2}, \cdots, h_{n}\right) \\
= & w_{1} h_{1} \oplus w_{2} h_{2} \oplus \cdots \oplus w_{n} h_{n} \\
= & \left\langle l_{\sum_{i=1}^{n} \omega_{n}\left(h_{i}\right) \eta\left(h_{i}\right)}, f^{-1}\left(\sum_{i=1}^{n} w_{i} f\left(u\left(h_{i}\right)\right)\right), g^{-1}\left(\sum_{i=1}^{n} w_{i} g\left(v\left(h_{i}\right)\right)\right)\right\rangle, \\
& \omega_{n}\left(h_{i}\right)=\frac{w_{i}\left[f\left(u\left(h_{i}\right)\right)+g\left(v\left(h_{i}\right)\right)\right]}{\sum_{i=1}^{n} w_{i}\left[f\left(u\left(h_{i}\right)\right)+g\left(v\left(h_{i}\right)\right)\right]},
\end{aligned}
$$


where $\left(w_{1}, w_{2}, \cdots, w_{n}\right)$ is the weight vector of ILNs $h_{i}=\left\langle l_{\eta\left(h_{i}\right)}, u\left(h_{i}\right), v\left(h_{i}\right)\right\rangle(i=1,2, \cdots, n)$, and $w_{i} \geq 0(i=1,2, \cdots, n), \sum_{i=1}^{n} w_{i}=1$, we then call ILWAA an intuitionistic linguistic weighted arithmetic averaging operator.

Theorem 3 (Commutativity) If $h_{i}^{\prime}(i=1,2, \cdots, n)$ is a permutation of $h_{i}(i=1,2, \cdots, n)$, then

$$
\operatorname{ILWAA}\left(h_{1}, h_{2}, \cdots, h_{n}\right)=\operatorname{ILWAA}\left(h_{1}^{\prime}, h_{2}^{\prime}, \cdots, h_{n}^{\prime}\right) .
$$

Proof By formula (22) and the condition that $h_{i}^{\prime}(i=1,2, \cdots, n)$ is a permutation of $h_{i}(i=1,2, \cdots, n)$, the result can be easily obtained.

Theorem 4 (Idempotency) If $h_{i}=h$ for all $i=1,2, \cdots, n$, then

$$
\operatorname{ILWAA}\left(h_{1}, h_{2}, \cdots, h_{n}\right)=h .
$$

Proof Let $h_{i}=h(i=1,2, \cdots, n)$, and $\sum_{i=1}^{n} w_{i}=1$, then by formula (22), we have $\operatorname{ILWAA}\left(h_{1}, h_{2}, \cdots, h_{n}\right)=h$.

Theorem 5 (Boundary) Let $h_{i}=\left\langle l_{\eta\left(h_{i}\right)}, u\left(h_{i}\right), v\left(h_{i}\right)\right\rangle(i=1,2, \cdots, n)$ be a collection of ILNs, and $h^{-}=\left\langle\min _{i}\left\{l_{\eta\left(h_{i}\right)}\right\}, \min _{i}\left\{u\left(h_{i}\right)\right\}, \max _{i}\left\{v\left(h_{i}\right)\right\}\right\rangle, h^{+}=\left\langle\max _{i}\left\{l_{\eta\left(h_{i}\right)}\right\}, \max _{i}\left\{u\left(h_{i}\right)\right\}, \min _{i}\left\{v\left(h_{i}\right)\right\}\right\rangle$, then

$$
h^{-} \leq \operatorname{ILWAA}\left(h_{1}, h_{2}, \cdots, h_{n}\right) \leq h^{+} .
$$

Proof Obviously,

$$
\begin{aligned}
& \min _{i}\left\{l_{\eta\left(h_{i}\right)}\right\} \preceq l_{\sum_{i=1}^{n} \omega_{n}\left(h_{i}\right) \eta\left(h_{i}\right)} \preceq \max _{i}\left\{l_{\eta\left(h_{i}\right)}\right\}, \\
& \min _{i}\left\{u\left(h_{i}\right)\right\} \leq f^{-1}\left(\sum_{i=1}^{n} w_{i}\left(f\left(u\left(h_{i}\right)\right)\right)\right) \leq \max _{i}\left\{u\left(h_{i}\right)\right\}, \\
& \min _{i}\left\{v\left(h_{i}\right)\right\} \leq g^{-1}\left(\sum_{i=1}^{n} w_{i} g\left(v\left(h_{i}\right)\right)\right) \leq \max _{i}\left\{v\left(h_{i}\right)\right\},
\end{aligned}
$$

then according to Definitions $9 \sim 11$, we have

$$
h^{-} \leq \operatorname{ILWAA}\left(h_{1}, h_{2}, \cdots, h_{n}\right) \leq h^{+} .
$$

\section{MADM Method Based on ILWAA Operator}

With respect to a MADM problem with intuitionistic linguistic information: Assume that $A=\left\{A_{1}, A_{2}, \cdots, A_{m}\right\}$ is a set of alternatives, and $G=\left\{G_{1}, G_{2}, \cdots, G_{n}\right\}$ is a set of attributes. Let the weight of the attribute $G_{j}$ be $w_{i}$, where $w_{i} \geq 0(i=1,2, \cdots, n)$, and $\sum_{i=1}^{n} w_{i}=1$. Let $R=\left(r_{i j}\right)_{m \times n}$ be the decision matrix, in which $r_{i j}=\left\langle l_{\eta\left(r_{i j}\right)}, u\left(r_{i j}\right), v\left(r_{i j}\right)\right\rangle$ denote an ILN, given by the decision maker for alternative $A_{i}$ with respect to attribute $G_{j}$. Then, we need to rank these alternatives and obtain the best alternative.

In the following we will employ the ILWAA operator to present a method to MADM with intuitionistic linguistic information. The method involves the following steps.

Step 1 Select the appropriate function $g$, then aggregate the intuitionistic linguistic information of alternative $A_{i}$ by ILWAA operator and get the comprehensive evaluation value $z_{i}(i=1,2, \cdots, m)$ 


$$
\begin{aligned}
& z_{i}=\operatorname{ILWAA}\left(r_{i 1}, r_{i 2}, \cdots, r_{i n}\right) \\
& =\left\langle l_{\sum_{j=1}^{n} w_{j}\left[f\left(u\left(r_{i j}\right)\right)+g\left(v\left(r_{i j}\right)\right)\right] \eta\left(r_{i j}\right)}, f^{-1}\left(\sum_{j=1}^{n} w_{j} f\left(u\left(r_{i j}\right)\right)\right), g^{-1}\left(\sum_{j=1}^{n} w_{j} g\left(v\left(r_{i j}\right)\right)\right)\right\rangle .
\end{aligned}
$$

Step 2 Calculate accumulated expectation $E\left(z_{i}\right)$ and accumulated score $S\left(z_{i}\right)$ of $A_{i}(i=$ $1,2, \cdots, m)$;

Step 3 Rank the alternative $A_{i}(i=1,2, \cdots, m)$ according to Definition 11, and then select the best one;

Step 4 End.

\section{Applying Example}

Suppose that an investment company intends to invest a sum of money. There are four possible alternatives $\left\{A_{1}, A_{2}, A_{3}, A_{4}\right\}$ for investing the money, the attributes are shown as follows: Management risk $\left(G_{1}\right)$, market risk $\left(G_{2}\right)$, production risk $\left(G_{3}\right)$, financial risk $\left(G_{4}\right)$. The four enterprises are to be evaluated with the ILS $\widetilde{H}=\left\{\left\langle l_{\eta}(x), u_{H}(x), v_{H}(x)\right\rangle \mid x \in X, l_{\eta}(x) \in \widetilde{L}_{6}\right\}$, where $L_{6}=\left\{l_{0}\right.$ (extremely poor), $l_{1}$ (very poor), $l_{2}$ (poor), $l_{3}$ (average), $l_{4}$ (good), $l_{5}$ (very good), $l_{6}$ (extremely good) $\}$. Assuming the weight vector of the four attributes is

$$
w=(0.30,0.25,0.3,0.15)^{\mathrm{T}} .
$$

The decision matrices are listed as follows:

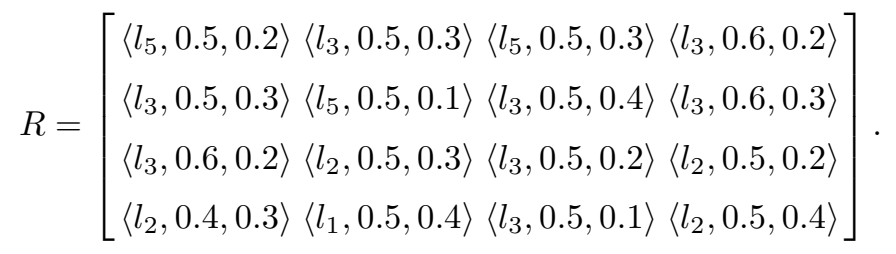

Because the attribute weight information is known, we can use the method introduced in Section 4. To obtain the selection results, the following steps are involved:

Step 1 Assume that the function $g(t)=-\log t$, and utilize the ILWAA operator to aggregate the attribute values, we can obtain the comprehensive aggregation results:

$$
\begin{aligned}
z_{i} & =\operatorname{ILWAA}\left(r_{i 1}, r_{i 2}, \cdots, r_{i n}\right) \\
& =\left\langle l_{\sum_{j=1}^{n} w_{j}\left[f\left(u\left(r_{i j}\right)\right)+g\left(v\left(r_{i j}\right)\right)\right] \eta\left(r_{i j}\right)}, f^{-1}\left(\sum_{j=1}^{n} w_{j} f\left(u\left(r_{i j}\right)\right)\right), g^{-1}\left(\sum_{j=1}^{n} w_{j} g\left(v\left(r_{i j}\right)\right)\right)\right\rangle, \\
z_{1} & =\left\langle l_{4} .2,0.52,0.25\right\rangle, \quad z_{2}=\left\langle l_{3} .7,0.51,0.25\right\rangle, \quad z_{3}=\left\langle l_{2} .6,0.53,0.22\right\rangle, \quad z_{4}=\left\langle l_{2} .2,0.47,0.24\right\rangle .
\end{aligned}
$$

Step 2 Calculate accumulated expectation $E\left(z_{i}\right)$ and accumulated score $S\left(z_{i}\right)$ of $A_{i}$,

$$
\begin{array}{lll}
E\left(z_{1}\right)=9.2, & Q\left(z_{1}\right)=4.7, \quad E\left(z_{2}\right)=8.1, & Q\left(z_{2}\right)=4.2 \\
E\left(z_{3}\right)=5.7, & Q\left(z_{3}\right)=3.3, \quad E\left(z_{4}\right)=3.7, & Q\left(z_{4}\right)=1.8
\end{array}
$$

Step 3 According to the ranking of accumulated expectation $E_{i}(i=1,2,3,4)$, the ranking is $A_{1}>A_{2}>A_{3}>A_{4}$. 


\section{Conclusions}

In a traditional document with intuitionistic linguistic numbers, the operational laws of theory are still imperfect. In order to overcome the blemish existed, the new operational laws combining Archimedean t-norm and its dual Archimedean s-norm, have been first defined, and a numerical example was given to illustrate the difference between these kind of operational laws. Later, we have studied some desirable properties of new operational laws, like commutativity, associativity and closure property etc. Then, we have developed an intuitionistic linguistic weighted arithmetic averaging (ILWAA) operator. Based on the ILWAA operator, a new method to multiple attribute decision making with intuitionistic linguistic information are proposed. At last, an illustrative example was given to verify the developed approaches and to demonstrate their practicality and effectiveness.

\section{References}

[1] Zadeh L A. Fuzzy sets versus probability. Proceedings of the IEEE, 1980, 68(3): 421-421.

[2] Cao Q W, Wu J. The extended COWG operators and their application to multiple attributive group decision making problems with interval numbers. Applied Mathematical Modelling, 2011, 35(5): 2075-2086.

[3] Yue Z. An extended TOPSIS for determining weights of decision makers with interval numbers. KnowledgeBased Systems, 2011, 24(1): 146-153.

[4] Cao Q W, Wu J. The extended COWG operators and their application to multiple attributive group decision making problems with interval numbers. Applied Mathematical Modelling, 2011, 35(5): 2075-2086.

[5] Parreiras R O, Ekel P Y, Martini J S C, et al. A flexible consensus scheme for multicriteria group decision making under linguistic assessments. Information Sciences, 2010, 180(7): 1075-1089.

[6] Alcalde C, Burusco A, Fuentes-Gonzlez R, et al. The use of linguistic variables and fuzzy propositions in the L-Fuzzy concept theory. Computers and Mathematics with Applications, 2011, 62(8): 3111-3122.

[7] Atanassov K T. Intuitionistic fuzzy sets. Fuzzy Sets and Systems, 1986, 20: 87-96.

[8] Atanassov K T. More on intuitionistic fuzzy sets. Fuzzy Sets and Systems, 1989, 33: 37-46.

[9] Atanassov K T. Operators over interval-valued intuitionistic fuzzy sets. Fuzzy Sets and Systems, 1994, 64: 159-174.

[10] Wang Z, Li K W, Wang W. An approach to multiattribute decision making with interval-valued intuitionistic fuzzy assessments and incomplete weights. Information Sciences, 2009, 179(17): 3026-3040.

[11] $\mathrm{Xu} \mathrm{Z} \mathrm{S.} \mathrm{Intuitionistic} \mathrm{preference} \mathrm{relations} \mathrm{and} \mathrm{their} \mathrm{application} \mathrm{in} \mathrm{group} \mathrm{decision} \mathrm{making.} \mathrm{Information}$ Sciences, 2007, 177(11): 2363-2379.

[12] Xu Z S. Models for multiple attribute decision making with intuitionistic fuzzy information. International Journal of Uncertainty Fuzziness and Knowledg-Based Systems, 2007, 15(3): 285-297.

[13] Atanassov K, Gargov G. Interval valued intuitionistic fuzzy sets. Fuzzy Sets and Systems, 1989, 31(3): 343-349.

[14] Shu M H, Cheng C H, Chang J R. Using intuitionistic fuzzy sets for fault-tree analysis on printed circuit board assembly. Microelectronics Reliability, 2006, 46(12): 2139-2148.

[15] Chen Y, Li B. Dynamic multi-attribute decision making model based on triangular intuitionistic fuzzy numbers. Scientia Iranica, 2011, 18(18): 268-274.

[16] Wang J Q, Li J J. The multi-criteria group decision making method based on multi-granularity intuitionistic two semantics. Science and Technology Information, 2009, 33: 8-9.

[17] Wang J Q. Multi-criteria interval intuitionistic fuzzy decision-making approach with incomplete certain information. Control and Decision 2006, 21(11): 1253-1256.

[18] Liu P D. Some generalized dependent aggregation operators with intuitionistic linguistic numbers and their application to group decision making. Journal of Computer and System Sciences, 2013, 79(11): 131-143.

[19] Liu P D, Jin F. Methods for aggregating intuitionistic uncertain linguistic variables and their application to group decision making. Information Sciences, 2012, 205(1): 58-71.

[20] Liu P D. Some geometric aggregation operators based on interval intuitionistic uncertain linguistic variables and their application to group decision making. Applied Mathematical Modelling, 2012, 37(4): 2430-2444. 
[21] Meng F, Chen X, Zhang Q. Some interval-valued intuitionistic uncertain linguistic Choquet operators and their application to multi-attribute group decision making. Applied Mathematical Modelling, 2014, 38(s 9-10): 2543-2557.

[22] Wang J Q, Li H B. Multi-criteria decision-making method based on aggregation operators for intuitionistic linguistic fuzzy numbers. Control and Decision 2010, 25(10): 1571-116.

[23] Liu P, Wang Y. Multiple attribute group decision making methods based on intuitionistic linguistic power generalized aggregation operators. Applied Soft Computing, 2014, 17(4): 90-104.

[24] Beliakov G, Pradera A, Calvo T. Aggregation Functions: A Guide for Practitioners. Studies in Fuzziness and Soft Computing, 2007, 221.

[25] Herrera F, Herrera-Viedma E, Verdegay J L. A model of consensus in group decision making under linguistic assessments. Fuzzy Sets and Systems, 1996, 79(1): 73-87.

[26] Herrera F, Herrera-Viedma E. Linguistic decision analysis: Steps for solving decision problems under linguistic information. Fuzzy Sets and Systems, 2000, 115(1): 67-82.

[27] Xu Z S. A method based on linguistic aggregation operators for group decision making with linguistic preference relations. Information Sciences, 2004, 166(s1-4): 19-30.

[28] Xu Z S. A note on linguistic hybrid arithmetic averaging operator in multiple attribute group decision making with linguistic information. Group Decision and Negotiation, 2006, 15(6): 593-604.

[29] Dai Y Q, Xu Z S. New evaluation scale of linguistic information and its application. Chinese Journal of Management Science, 2008, 16(2): 145-149. 